\title{
Treatment of Pyonephritis Complicated by Sepsis-Induced Disseminated Intravascular Coagulation Using Recombinant Human Soluble Thrombomodulin and Percutaneous Drainage
}

\author{
Nobuhiro Takeuchia, c, Kazumasa Emori ${ }^{\text {a }}$, Makoto Yoshitani ${ }^{a}$, Junichi Soneda ${ }^{\text {b }}$
}

\begin{abstract}
Pyonephritis is defined as infected hydronephrosis complicated by infectious destruction of the renal parenchyma or the presence of pus in the dilated pyelocaliceal. Pyonephritis is challenging to treat only using antibiotics; it sometimes requires immediate interventional treatment, including surgical intervention, percutaneous drainage, or retrograde ureteric stent insertion, because if left untreated, it may cause sepsisrelated disseminated intravascular coagulation (DIC). Here we report three cases of pyonephritis complicated by sepsis-induced DIC using recombinant human soluble thrombomodulin (rhTM) and percutaneous drainage. An 87-year-old female presented with high-grade fever, vomiting, and diarrhea. Non-contrast computed tomography and ultrasonography revealed a dilated ureter and upper ureteral stone in the left kidney. Antibiotic treatment was initiated, and a percutaneous nephrostomy (PCN) tube was inserted into the patient; puncture fluid revealed the presence of Escherichia coli. During treatment, the patient's DIC score was four points, indicating that rhTM administration was required. A 75-year-old female presented with nausea, diarrhea, and deterioration of consciousness. Laboratory analysis revealed remarkably elevated levels of WBC and CRP, and imaging modalities revealed left-sided hydronephrosis. The patient's DIC score at admission was six points; rhTM and anti-biotics were administered. A PCN tube was inserted into the patient on the following day. With worsening renal function and oliguria, the patient needed hemodialysis (polymyxin-B direct hemoperfusion and continuous hemodiafiltration). A 62-year-old female presented with high-grade fever and deterioration of consciousness. The patient's DIC score at admission was four points; rhTM and antibiotics were administered. Imaging modalities showed left-sided hydronephrosis and, the patient required PCN tube insertion. Temporal hemodialysis alleviated the patient's renal malfunction and septicemia. All three cases recovered from sepsis-related DIC. Immediate and intensive treatments for pyonephritis, including interventional drainage,
\end{abstract}

Manuscript accepted for publication November 15, 2016

aDepartment of Internal Medicine, Kobe Tokushukai Hospital, 1-3-10 Kamitakamaru, Tarumi-ku, Kobe-shi, Hyogo 655-0017, Japan

${ }^{b}$ Department of Cardiovascular Surgery, Kobe Tokushukai Hospital, 1-3-10 Kamitakamaru, Tarumi-ku, Kobe-shi, Hyogo 655-0017, Japan

${ }^{\mathrm{c} C o r r e s p o n d i n g ~ A u t h o r: ~ N o b u h i r o ~ T a k e u c h i, ~ D e p a r t m e n t ~ o f ~ I n t e r n a l ~ M e d i-~}$ cine, Kobe Tokushukai Hospital, 1-3-10 Kamitakamaru, Tarumi-Ku, Kobeshi, Hyogo, 655-0017, Japan. Email: nobuhiro.takeuchi@tokushukai.jp

doi: https://doi.org/10.14740/wjnu283w hemodialysis, administration of antibiotics, and the management of DIC, are necessary to save patients from fatal conditions.

Keywords: Pyonephritis; Sepsis; Disseminated intravascular coagulation

\section{Introduction}

Thrombomodulin is an integral membrane protein expressed on the surface of endothelial cells that plays a role as a thrombin co-factor. The complex formed between thrombin and thrombomodulin (the thrombin-thrombomodulin complex) inhibits fibrinolysis by activating protein $\mathrm{C}$ (activated protein $\mathrm{C}$ (APC)). APC inhibits the production of thrombin by dissolving activated V and VII factors, exhibits anti-inflammatory and anti-fibrinolytic effects, and is deemed to be effective for sepsis-related disseminated intravascular coagulation (DIC). Recombinant human soluble thrombomodulin (rhTM) is a novel class of anticoagulants used for the treatment of DIC, and is becoming a mainstay for DIC management. The structure of rhTM consists of the active and extracellular domains of thrombomodulin on the endothelial surface, and works similarly to thrombomodulin by binding to thrombin and, activating APC.

Pyonephritis is defined as infected hydronephrosis complicated by an infectious destruction of the renal parenchyma, or the pus-filled dilated pyelocaliceal. Pyonephritis is challenging to treat only with anti-bacterial drugs and it can require immediate interventional treatment, including surgical intervention, percutaneous drainage, or retrograde ureteric stent insertion. This is because if left untreated, DIC-related septicemia can ensue.

In this study, we present three cases of pyonephritis complicated by DIC that were successfully treated using rhTM and the insertion of a percutaneous nephrostomy (PCN) tube.

\section{Case Reports}

\section{Case 1}

An 87-year-old female visited our hospital with a high-grade fever, nausea, and diarrhea. Her past medical history included 

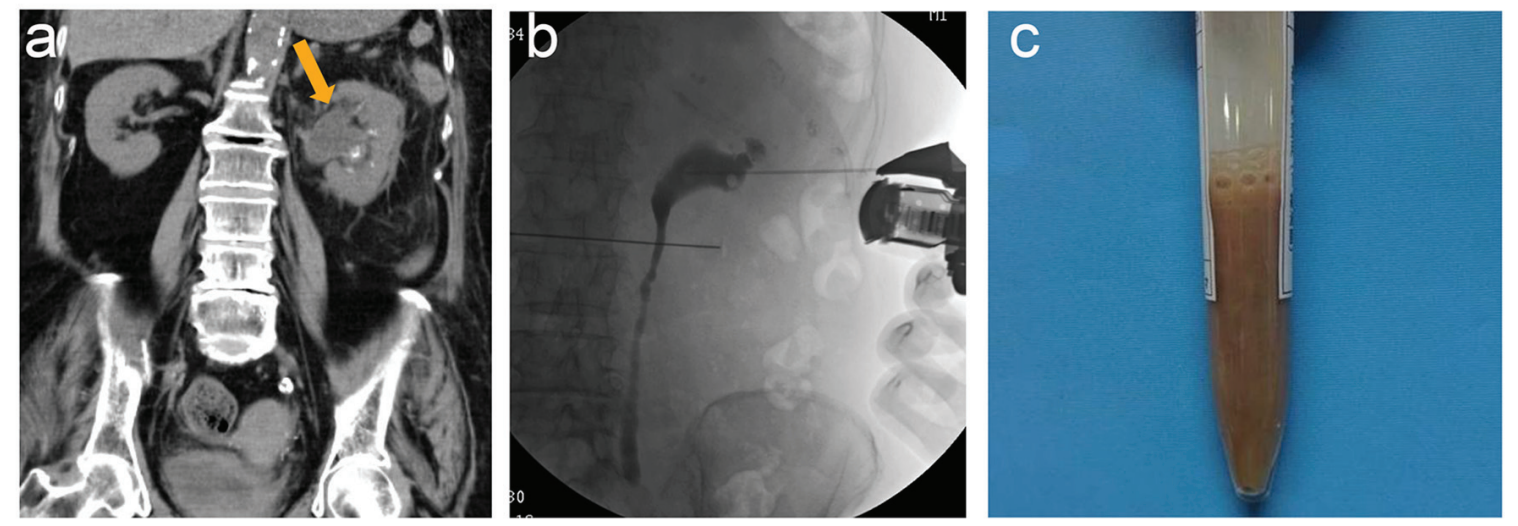

Figure 1. (a) Non-contrast computed tomography revealed a dilated left ureter and swollen left kidney with fat stranding in the peritoneal space. (b) A percutaneous nephrostomy tube was inserted under ultrasonography, revealing a dilated ureter and renal pelvis. (c) The drainage contained brown pus with a foul odor.

cerebral dementia, type 2 diabetes mellitus, and dyslipidemia. The physical examination upon her arrival revealed that her blood pressure was $100 / 72 \mathrm{~mm} \mathrm{Hg}$, heart rate was 100 beats/ min with a regular rhythm, blood oxygen saturation was $97 \%$ under atmospheric conditions, and her body temperature was $39.5{ }^{\circ} \mathrm{C}$. Moreover, she exhibited a disturbed consciousness disturbance with a II-30 score of the Japan coma scale (JCS). The blood analysis revealed a reduced number of white blood cells (WBCs 2,110 cells $/ \mu \mathrm{L}$ ), mild hypoproteinemia (6.7 g/ $\mathrm{dL})$, severe hypoalbuminemia ( $2.5 \mathrm{~g} / \mathrm{dL})$, and impaired glucose tolerance $(208 \mathrm{mg} / \mathrm{dL}$ glucose and $7.6 \%$ hemoglobin A1c). The urinalysis revealed more than $100 \mathrm{WBCs} / \mathrm{HPF}, 30$ - 49/HPF red blood cells (RBCs), as well as severe proteinuria. Non-contrast computed tomography $(\mathrm{CT})$ revealed a dilated left ureter, swollen left kidney with fat stranding in the retroperitoneal space, and an incarcerated stone in the upper left ureter (Fig. 1a). The patient was diagnosed with infective hydronephrosis, and was subsequently admitted to our hospital for further examination and treatment. The administration of flomoxef sodium at a dose of $2 \mathrm{~g} /$ day was initiated. The high-grade fever continued, and then her serum platelet levels dropped from $12.6 \times 10^{4}$ to $11.2 \times 10^{4}$ cells $/ \mu \mathrm{L}$ on the following day. On day 3 , the high-grade fever continued and the laboratory analyses revealed remarkably elevated $\mathrm{C}$-reactive protein levels $(28.92 \mathrm{mg} / \mathrm{dL})$, drastically elevated procalcitonin levels $(>100 \mathrm{ng} / \mathrm{mL})$, further reduced platelet levels $\left(9.7 \times 10^{4}\right.$ cells/ $\mu \mathrm{L}$ ), and deteriorated renal function (creatinine levels $1.14 \mathrm{mg}$ / $\mathrm{dL}$; blood urea nitrogen $23.6 \mathrm{mg} / \mathrm{dL}$ ). Moreover, the urine and blood culture obtained upon admission was positive for Escherichia coli. At this time, abscess drainage of the left pelvis

$\mathrm{PLT} \times 10^{4} / \mu \mathrm{L}$

$\mathrm{FDP} \mu \mathrm{g} / / \mathrm{mL}$

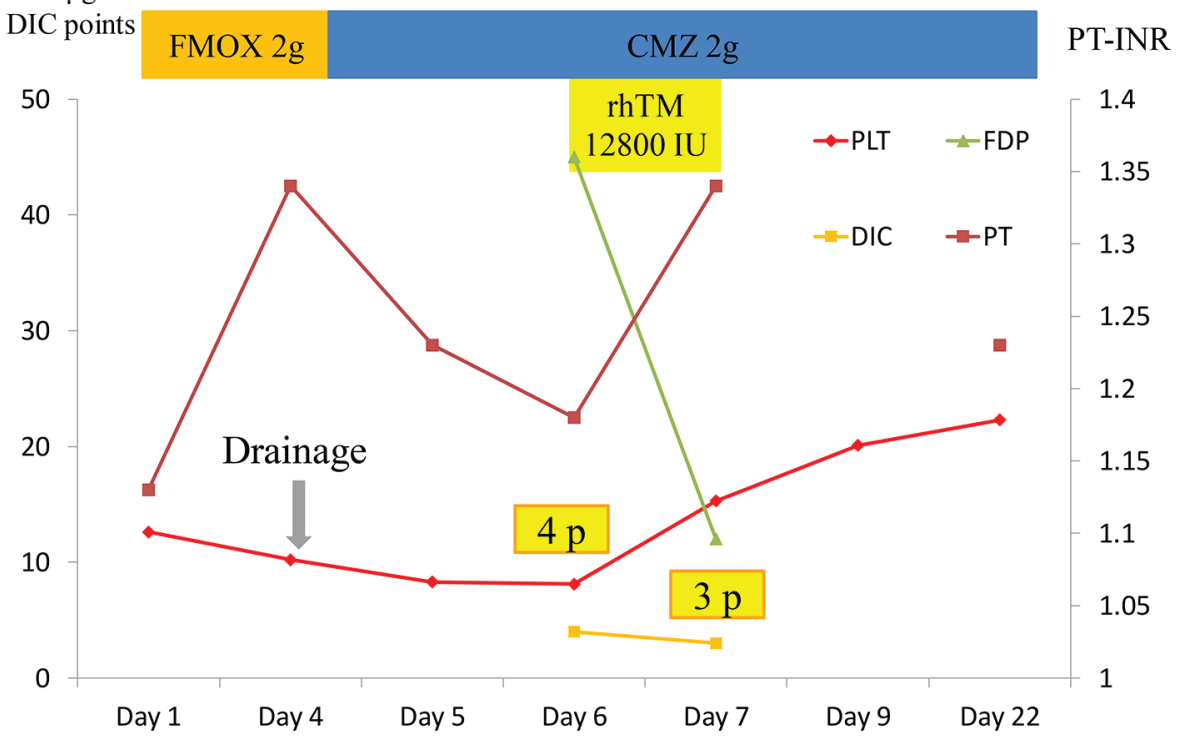

Figure 2. The clinical course of case 1. FMOX: flomoxef; CMZ: cefmetazole. 


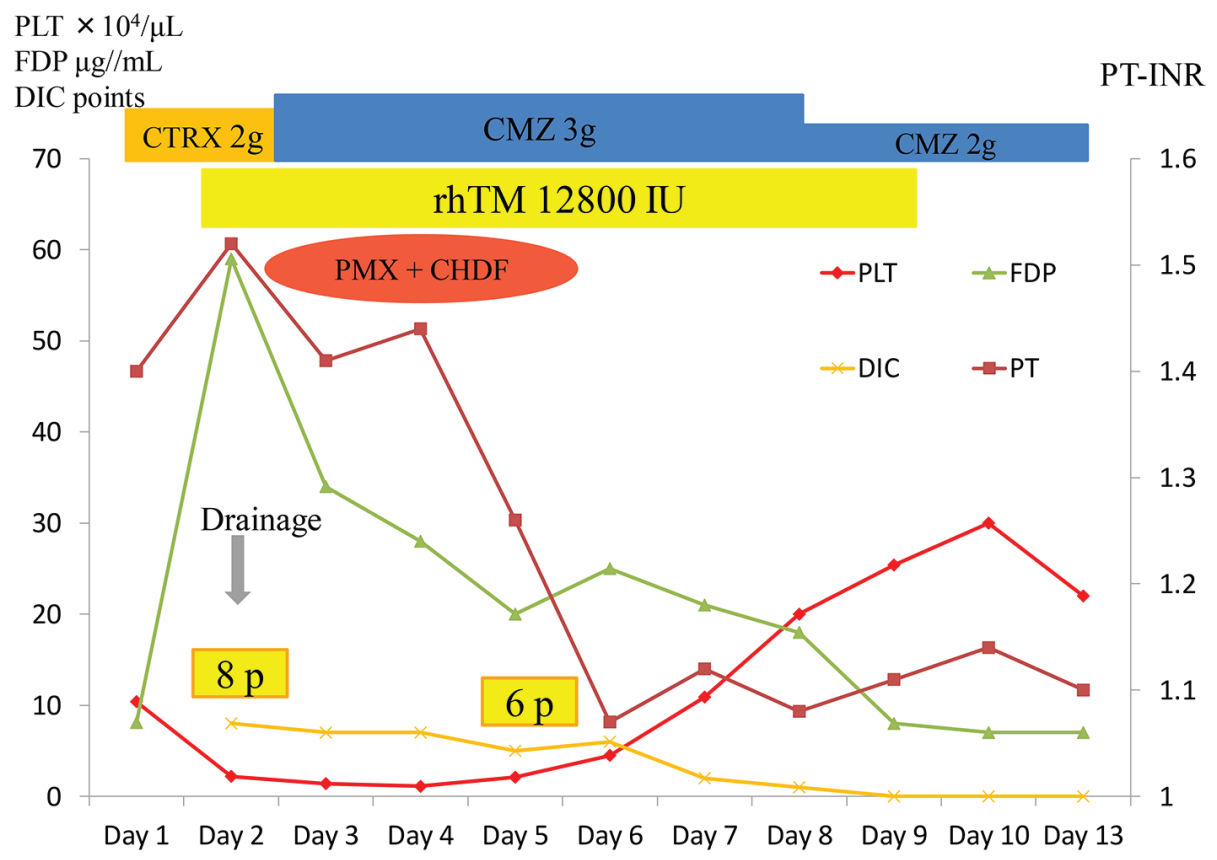

Figure 3. The clinical course of case 2. CTRX: ceftriaxone; CMZ: cefmetazole.

was scheduled to control the infection and a percutaneous nephrostomy (PCN) tube was inserted (Fig. 1b). The drainage contained brown pus with a foul odor (Fig. 1c). The drainage culture revealed positive results for $E$. coli, which was sensitive to cefmetazole (CMZ). The antibiotic drug was changed to $\mathrm{CMZ}$ at a dose of $3 \mathrm{~g} /$ day. On day 6 , the WBC count dropped to 3,420 cells $/ \mu \mathrm{L}$, fibrin degradation products (FDP) were elevated to $45 \mu \mathrm{g} / \mathrm{mL}$, and the platelet count was $8.1 \times 10^{4}$ cells/ $\mu \mathrm{L}$, which was consistent with a DIC score of four points. An administration of 12,800 IU rhTM was initiated and continued for 3 days until the DIC score dropped to three points. Moreover, there was an improvement in the inflammatory reaction, platelet levels, and coagulant disturbance. The clinical course was shown in Figure 2.

\section{Case 2}

A 75-year-old female presented with nausea, diarrhea, and a disturbance of consciousness. The patient's past medical history included type 2 diabetes mellitus which was treated with regular subcutaneous insulin infusion. The physical examination upon arrival revealed that her blood pressure was 130/70 $\mathrm{mm} \mathrm{Hg}$, heart rate was 80 beats/min with a regular rhythm, blood oxygen saturation was $93 \%$ under $3 \mathrm{~L} / \mathrm{min}$ oxygen inhalation, and her body temperature was $37.3^{\circ} \mathrm{C}$. A disturbance of consciousness was evident with a JCS rating of II-30. Moreover, laboratory analyses revealed severe inflammatory reactions $(30,860 \mathrm{WBCs} / \mu \mathrm{L}$ comprised of $96.2 \%$ neutrophils and $12.77 \mathrm{mg} / \mathrm{dL}$ C-reactive protein), severe hypoproteinemia (4.8 $\mathrm{g} / \mathrm{dL})$, severe hypoalbuminemia $(2.4 \mathrm{~g} / \mathrm{dL})$, mild hyponatremia $(129 \mathrm{mEq} / \mathrm{mL})$, severely impaired glucose tolerance $(224 \mathrm{mg} /$ dL glucose and $11.4 \%$ hemoglobin A1c), and a mild coagula- tion disorder (98\% prothrombin and $32.7 \mathrm{~s}$ activated partial thromboplastic time). The urinalysis revealed more than 100 WBCs/HPF, 30 - 49/HPF RBCs, and severe proteinuria. Noncontrast CT and USG revealed left-sided hydronephrosis, and she was diagnosed with infective hydronephrosis. Immediately following admission, ceftriaxone (CTRX) was initiated at a dose of $2 \mathrm{~g} /$ day. On the following day, the platelet counts dropped from $19.4 \times 10^{4}$ to $10.4 \times 10^{4}$ cells $/ \mu \mathrm{L}$. Furthermore, the laboratory analyses revealed a worsening renal function $(2.9 \mathrm{mg} / \mathrm{dL}$ Cre and $52.1 \mathrm{mg} / \mathrm{dL}$ BUN) and a coagulation disorder (FDP $81 \mu \mathrm{g} / \mathrm{mL}$ and PT-INR 1.40). The diagnosis of DIC was eight points, and the administration of rhTM at a dose of $128,000 \mathrm{IU} /$ day was initiated. The blood and urine cultures obtained at the time of arrival revealed the presence of $E$. coli which was sensitive to CMZ. In accordance with these results, the antibiotics were changed to $\mathrm{CMZ}$ at a dose of $3 \mathrm{~g} /$ day. On the same day, a PCN tube was inserted into the patient and pus culture was positive for E. coli. On day 3, due to oliguria, deteriorated renal function, and impaired circulatory function, hemodialysis (polymyxin-B direct hemoperfusion and continuous hemodiafiltration) was initiated for three consecutive days. Subsequently, her circulatory condition stabilized, and the DIC score decreased to six points on day 6 and further improved to two points on day 7 . The clinical course was uneventful, and she was discharged. The clinical course was shown in Figure 3.

\section{Case 3}

A 62-year-old female presented with a high-grade fever and disturbance of consciousness. Her medical history included type II diabetes mellitus which was treated using oral antidiabetic drugs and rheumatoid arthritis treated with prednisolone 


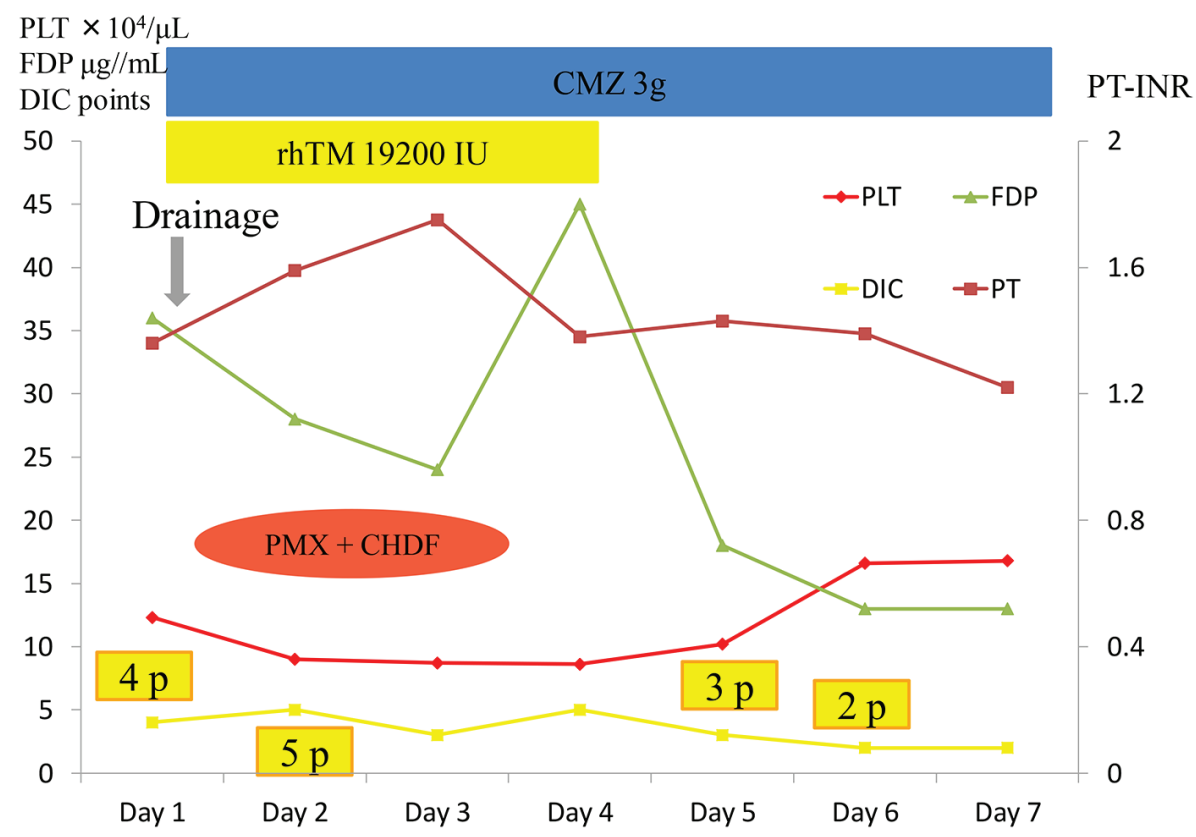

Figure 4. The clinical course of case 3. CMZ: cefmetazole.

at a dose of $5 \mathrm{mg} /$ day. Upon arrival, her consciousness was rated as JCS II-10, her blood pressure was 110/64 mm Hg, heart rate was 87 beats/min with a regular rhythm, blood oxygen saturation was $96 \%$ under atmospheric air, and her body temperature was $39.6{ }^{\circ} \mathrm{C}$. Inspection of the palpebral conjunctiva revealed mild anemia. The abdomen was soft and flat, and no tenderness was observed. Moreover, the laboratory analyses revealed moderate anemia $\left(243 \times 10^{4} \mathrm{RBCs} / \mu \mathrm{L}, 8.4 \mathrm{~g} / \mathrm{dL}\right.$ hemoglobin, and $25.2 \%$ hematocrit), mild hypoproteinemia (6.2 $\mathrm{g} / \mathrm{dL})$, severe hypoalbuminemia $(2.3 \mathrm{~g} / \mathrm{dL})$, mildly elevated liver and biliary enzymes (110 IU/L gamma-glutamyltransferase, $498 \mathrm{IU} / \mathrm{L}$ alkaline phosphatase, $68 \mathrm{IU} / \mathrm{L}$ AST, and 45 IU/L ALT), renal dysfunction $(1.98 \mathrm{mg} / \mathrm{dL}$ Cre and $36.5 \mathrm{mg} / \mathrm{dL}$ BUN), mild hyponatremia $(125 \mathrm{mEq} / \mathrm{mL})$, severely impaired glucose tolerance $(449 \mathrm{mg} / \mathrm{dL}$ glucose and $6.9 \%$ hemoglobin A1c), severely elevated C-reactive proteins $(24.22 \mathrm{mg} / \mathrm{dL})$, and a mild coagulation disorder (67\% prothrombin, $453 \mathrm{mg} /$ $\mathrm{dL}$ fibrinogen, and $36 \mu \mathrm{g} / \mathrm{mL}$ FDP). The urinalysis revealed an increase in WBCs $>100 / \mathrm{HPF}$, increase in RBCs, mild proteinuria, and elevated urinary sugar. Both CT and USG revealed left-sided hydronephrosis. Based on the laboratory analyses and imaging modalities, pyonephrosis was suspected, and a PNC tube was inserted into the patient. Upon admission, the DIC score was four points and the administration of rhTM at a dose of 192,000 IU/day was initiated in conjunction with CMZ at a dose of $3 \mathrm{~g} /$ day. The pus, blood, and urine culture revealed the presence of Klebsiella pneumoniae that was sensitive to CMZ. On the following day, the DIC score worsened to five points and due to decreased renal function, circulatory insufficiency, and the presence of oliguria, the patient required a continuous administration of noradrenaline and PMX-DHP + CHDF. RhTM was continued until day 5 when the DIC score improved to three points. For this patient, immediate manage- ment of DIC, percutaneous drainage, and hemodialysis was effective for improving her condition. The clinical course was shown in Figure 4.

\section{Discussion}

Urosepsis is the most severe condition that develops as the result of a urinary tract infection and can be further complicated by DIC, leading to high morbidity. Pyonephritis is one form of a urinary tract infection that is accompanied by the condition of pus under pressure, may deteriorate rapidly and fall into sepsis or sepsis-induced DIC. Therefore, immediate intervention is required for this fatal condition using intensive approaches, including the administration of antibacterial drugs, percutaneous drainage, the insertion of a retrograde ureteric stent ins, or management of DIC.

It is known that the typical symptoms of pyonephritis consist of fever, back pain, and a palpable mass [1]; however, in some cases, the above symptoms are absent and the patient will present only with a fever and gastrointestinal symptoms. The cause of pyonephritis in the majority of cases is a ureter stone $(70 \%)$, while $10 \%$ of causes are unknown. In our cases, the patients presented with a fever and gastrointestinal symptoms; thus, the diagnosis of pyonephritis could not be made without the results of various imaging modalities, laboratory analyses, and cultures. The primary method of treatment for pyonephritis is drainage, and if it left untreated, increased pressure in the infected pelvis might progress to septicemia. Whenever the clinical manifestation and CT findings suggest the presence of pyonephritis, immediate drainage is recommended to protect renal function. A recent report surveying the effect of surgical drainage in infective hydronephrosis demonstrated decreased 
mortality in patients following surgical decompression $(8.82 \%$ mortality in the surgical decompression group vs. $19.2 \%$ in the conservative therapy group) [2]. Regarding the methods of surgical decompression, it remains controversial whether a PCN tube or ureter stent placement is favorable. A PCN tube placement is reported to yield a shorter amount of time for alleviating symptoms and a better quality of life compared to a ureter stent placement; however, there is a $5 \%$ risk of major complications $[3,4]$.

Conventional DIC treatment consists of anticoagulant therapy, including nafamostat mesilate, gabexate mesilate, heparin sodium, and antithrombin concentration, as well as the replacement of coagulation factors and platelet preparation. Recent DIC treatment has focused on suppressing the inflammatory activity of WBCs or cytokines. Therefore, the use of rhTM for its anti-coagulation and anti-inflammatory effects has been added to DIC management.

RhTM is a novel class of anticoagulants used for the treatment of DIC, and is becoming a mainstay for DIC management. In Japan, rhTM (Asahi Kasei Pharm Co., Tokyo, Japan) was approved in 2008 and has subsequently been widely used for the treatment of DIC caused by various underlying diseases, including infections, solid and blood malignancies, and aortic disease. Moreover, rhTM is known to have a powerful anticoagulant effect, and there have been several reports describing a tendency for increased bleeding due to the use of rhTM, including cerebral and pulmonary bleeding [5]. However, a recent systemic review reported that there was no significant difference regarding bleeding complications between patients treated with or without rhTM [6]. Nonetheless, careful attention is required regarding the use of rhTM in severe cases due to the anticoagulant effect of rhTM. In our cases, there were no serious bleeding complications. In the Japanese guidelines for the management of sepsis, the recommendation for using rhTM, antithrombin III preparation, and low molecular weight heparin in the setting of DIC is ranked as 2C [7]. The administration of an antithrombin III preparation is considered to improve DIC; however, in clinical settings, it is not used due to the lack of data demonstrating an improvement in mortality [8].

In the pathogenesis of sepsis, the cross-talk between the inflammatory and coagulation responses play an important role via high-mobility group box 1 (HMGB-1), which can lead to the development of DIC and multiple organ failure (MOF) [9]. The lectin-like domain of TM plays an important role in suppressing inflammation independent of TM anticoagulant activity. In addition, it directly exerts an anti-inflammatory effect by binding and inactivating HMGB-1 [10]. In the management of sepsis, a treatment strategy employed to reduce HMGB-1 levels is crucial; thus, PMX-DHP is expected to be an effective method as it indirectly decrease HMGB-1 via a decrease in serum cytokine levels, including interleukin-6. This is because it absorbs endotoxin, as well as activated monocytes and endogenous cannabinoids [11]. In contrast, it has been suggested that the polymyxin B-immobilized fiber column does not have an effective ability to absorb HMGB-1 due to clinical experiences and basic medicine. Thus, in cases of severe sepsis complicated by DIC and the presence of elevated serum HMGB-1 levels, single therapy with only PMX-DHP may not yield high survival rates. Moreover, the polymyxin B-immobilized fiber column does not absorb rhTM; thus, in the combination therapy of PMH-DHP and rhTM, the effects of rhTM cannot be weakened. Furthermore, during sepsis, the serum levels of TM are decreased and thus, the administration of rhTM is considered to be reasonable. A recent study involving patients with septic shock accompanied by DIC was associated with higher survival rates for the combination therapy of PMX-DHP and rhTM compared to the single therapy using PMX-DHP [12]. The combination therapy of PMH-DHP and CHDFP has been proven to ameliorate renal function in patients with sepsis [13]; moreover, such therapy is suggested to have an effect on deducing the circulating levels of HMGB-1 [14]. In our cases (cases 2 and 3), intensive therapy combined with rhTM, percutaneous drainage, and PMX-DHP + CHDF, was effective for improving sepsis-related DIC and deteriorated renal function.

\section{Conclusion}

In this study, we reported three cases of pyonephritis complicated by DIC that were successfully treated using rhTM and a percutaneous PCN tube insertion. Pyonephritis is a critical condition that may lead to septicemia and DIC. Treatment of pyonephritis via drainage, whether it is attempted percutaneously or under a cystoscope, is required to control and improve infections that are associated with abscess formation. With the introduction of rhTM, DIC treatment is expected to undergo a paradigm shift. In septic-related DIC, the use of rhTM with or without PMH-DHP may become the key to improving critical conditions without imposing a serious risk of bleeding.

\section{Acknowledgments}

The authors would like to thank Kaori Mohri and Kumiko Kawabuchi for their support to this manuscript.

\section{Consent}

The patients gave written informed consent for publication of this case report and all accompanying images.

\section{Competing Interests}

The authors declare that they have no competing interests.

\section{References}

1. Androulakakis PA. Pyonephrosis: a critical review of 131 cases. Br J Urol. 1982;54(2):89-92.

2. Borofsky MS, Walter D, Shah O, Goldfarb DS, Mues AC, Makarov DV. Surgical decompression is associated with decreased mortality in patients with sepsis and ureteral calculi. J Urol. 2013;189(3):946-951. 
3. Ramsey S, Robertson A, Ablett MJ, Meddings RN, Hollins GW, Little B. Evidence-based drainage of infected hydronephrosis secondary to ureteric calculi. J Endourol. 2010;24(2):185-189.

4. Mokhmalji H, Braun PM, Martinez Portillo FJ, Siegsmund M, Alken P, Kohrmann KU. Percutaneous nephrostomy versus ureteral stents for diversion of hydronephrosis caused by stones: a prospective, randomized clinical trial. J Urol. 2001;165(4):1088-1092.

5. Saito H, Maruyama I, Shimazaki S, Yamamoto Y, Aikawa N, Ohno R, Hirayama A, et al. Efficacy and safety of recombinant human soluble thrombomodulin (ART-123) in disseminated intravascular coagulation: results of a phase III, randomized, double-blind clinical trial. J Thromb Haemost. 2007;5(1):31-41.

6. Yamakawa K, Aihara M, Ogura H, Yuhara H, Hamasaki T, Shimazu T. Recombinant human soluble thrombomodulin in severe sepsis: a systematic review and metaanalysis. J Thromb Haemost. 2015;13(4):508-519.

7. Oda S, Aibiki M, Ikeda T, Imaizumi H, Endo S, Ochiai R, Kotani J, et al. The Japanese guidelines for the management of sepsis. J Intensive Care. 2014;2(1):55.

8. Warren BL, Eid A, Singer P, Pillay SS, Carl P, Novak I, Chalupa $\mathrm{P}$, et al. Caring for the critically ill patient. Highdose antithrombin III in severe sepsis: a randomized controlled trial. JAMA. 2001;286(15):1869-1878.
9. Rittirsch D, Flierl MA, Ward PA. Harmful molecular mechanisms in sepsis. Nat Rev Immunol. 2008;8(10):776787.

10. Ito $\mathrm{T}$, Kawahara $\mathrm{K}$, Okamoto $\mathrm{K}$, Yamada $\mathrm{S}$, Yasuda M, Imaizumi H, Nawa Y, et al. Proteolytic cleavage of high mobility group box 1 protein by thrombin-thrombomodulin complexes. Arterioscler Thromb Vasc Biol. 2008;28(10):1825-1830.

11. Ueno T, Ikeda T, Ikeda K, Taniuchi H, Suda S, Yeung MY, Matsuno N. HMGB-1 as a useful prognostic biomarker in sepsis-induced organ failure in patients undergoing PMX-DHP. J Surg Res. 2011;171(1):183-190.

12. Yamato M, Minematsu Y, Fujii J, Mori K, Minato T, Miyagawa S, Fujimura R, et al. Effective combination therapy of polymyxin-B direct hemoperfusion and recombinant thrombomodulin for septic shock accompanied by disseminated intravascular coagulation: a historical controlled trial. Ther Apher Dial. 2013;17(5):472-476.

13. Mitaka C, Masuda T, Kido K, Uchida T, Abe S, Miyasho T, Tomita M, et al. Polymyxin B hemoperfusion prevents acute kidney injury in sepsis model. J Surg Res. 2016;201(1):59-68.

14. Ueno T, Ikeda T, Yokoyama T, Kihara Y, Konno O, Nakamura Y, Iwamoto H, et al. Reduction in circulating level of HMGB-1 following continuous renal replacement therapy in sepsis. Cytokine. 2016;83:206-209. 